\title{
Structural and Optical Properties of Boron Nitride Grown by MOVPE
}

\author{
A.K. Dąbrowska, K. PakuŁa, R. Bożek, J.-G. Rousset, D. ZióŁkowska, K. Goøasa, \\ K.P. Korona, A. WysmoŁek And R. StęPnieWski \\ Institute of Experimental Physics, Faculty of Physics, University of Warsaw, L. Pasteura 5, 02-093 Warsaw, Poland
}

\begin{abstract}
Boron nitride layers were grown on sapphire substrate by metal organic vapor phase epitaxy system that was originally designed for growth of GaN. Structures were characterized by scanning electron microscopy, atomic force microscopy, the Raman spectroscopy, absorption and time resolved photoluminescence. Presented results confirm successful deposition of BN layers and gives information about basic properties of the material. The Raman line at $1370 \mathrm{~cm}^{-1}$ and absorption edges at 5.6-5.9 eV were observed which is related to hexagonal phase.
\end{abstract}

DOI: 10.12693/APhysPolA.129.A-129

PACS: 81.15.Gh, 78.30.Fs, 78.20.-e

\section{Introduction}

Boron nitride is a wide band gap III-V compound with unique physical and chemical properties. It may crystallize in three structures: cubic, hexagonal (graphite-like) and wurtzite [1]. For electronic application, most promising is hexagonal boron nitride (h-BN), with structural properties similar to graphite and graphene. There are two main potential applications of h-BN. The first one is related to a conventional application for semiconductor compounds, such as light source in the deep ultraviolet [2]. The second one is strongly associated with development of two-dimensional materials. Stacked structures of graphene, h-BN, $\mathrm{MoS}_{2}$ etc. lead to formation of new materials with interesting properties [3]. In this context, there is a need to better understand this material in terms of its fundamental properties.

Technology of h-BN thin layer deposition encounter some specific problems and is still on initial stage of development. In metal organic vapor phase epitaxy (MOVPE) method, strong gas-phase parasitic reactions cause extremely low efficiency of the growth. Lack of appropriate substrates and low on-surface diffusion result in weak long range ordering and grainy structure of the layers.

In this paper we present results of preliminary studies of BN layers obtained during technological work on growth efficiency optimisation. Structural and optical investigations were performed giving information about fundamental properties of the material.

\section{Experiment and samples}

Boron nitride layers were grown by MOVPE method, using reactor originally designed for growth of GaN. The $c$-plane, 2" diameter sapphire was used as substrates. $\mathrm{BN}$ was deposited directly on sapphire or was preceded by standard GaN layer, to check influence on BN nucleation. Deposition conditions were similar to that used for GaN. Some variations of growth parameters were used to increase the growth efficiency. Growth rate was monitored in situ using interferometer. Maximum growth rate of $150 \mathrm{~nm}$ per hour was achieved.
Structural and optical properties of the layers were investigated. The thickness, homogeneity and surface morphology were examined by optical and scanning electron microscopy (SEM). Additionally atomic force microscopy (AFM) was used. The Raman scattering was measured with Renishaw spectrometer. Absorption spectra was measured by UV-Vis-NIR Cary 5000 spectrometer at room temperature. For time resolved photoluminescence a Hamamatsu streak camera was used.

\section{Results and discussion}

\subsection{Structural properties}

Final BN layers had thickness from $50 \mathrm{~nm}$ to $300 \mathrm{~nm}$ with good uniformity on whole substrates surface. Monochrome light interference on the thicker layers was observed with naked eye. This effect was reduced by surface roughness visible in optical microscopy with maximum magnifications, in a scale below $1 \mu \mathrm{m}$. Observation with AFM confirms roughness of the surface and complex structure of the layers.

Observation performed with SEM revealed grainy structure of the BN layers. Figure 1a shows the edge of intentionally broken sample. Sapphire substrate is covered with $300 \mathrm{~nm}$ thick BN layer. The grains of BN are clearly distinguished. Roughness of the surface morphology is a direct result of such internal structure.

Grainy structure of BN layers is a result of weak, rare nucleation. The growth starts from separated sites with distance of hundreds nm. Grains slowly increase, connect each other and form continuous layer. It is process similar to that observed in GaN growth on sapphire. BN layers deposited directly on sapphire are relatively easy to scrap off. Mechanically scrapped-off layer chips have diameter tens time larger than layer thickness. Example of such destroyed layer is shown in Fig. 1b.

In the case of $\mathrm{BN}$ deposited on $\mathrm{GaN} /$ sapphire substrate, spontaneous ex-foliation of BN layer was observed for layer with thickness exciting $300 \mathrm{~nm}$ (Fig. 1c). This exfoliation probably starts during cooling down, after the 
growth process. Largest lifted-off parts of BN layer had a diameter of $1 \mathrm{~mm}$. These parts could be easily taken from substrate and investigated separately.

Weak bonds between BN layer and substrates can be direct result of hexagonal, graphite-like crystal structure of $\mathrm{BN}$.

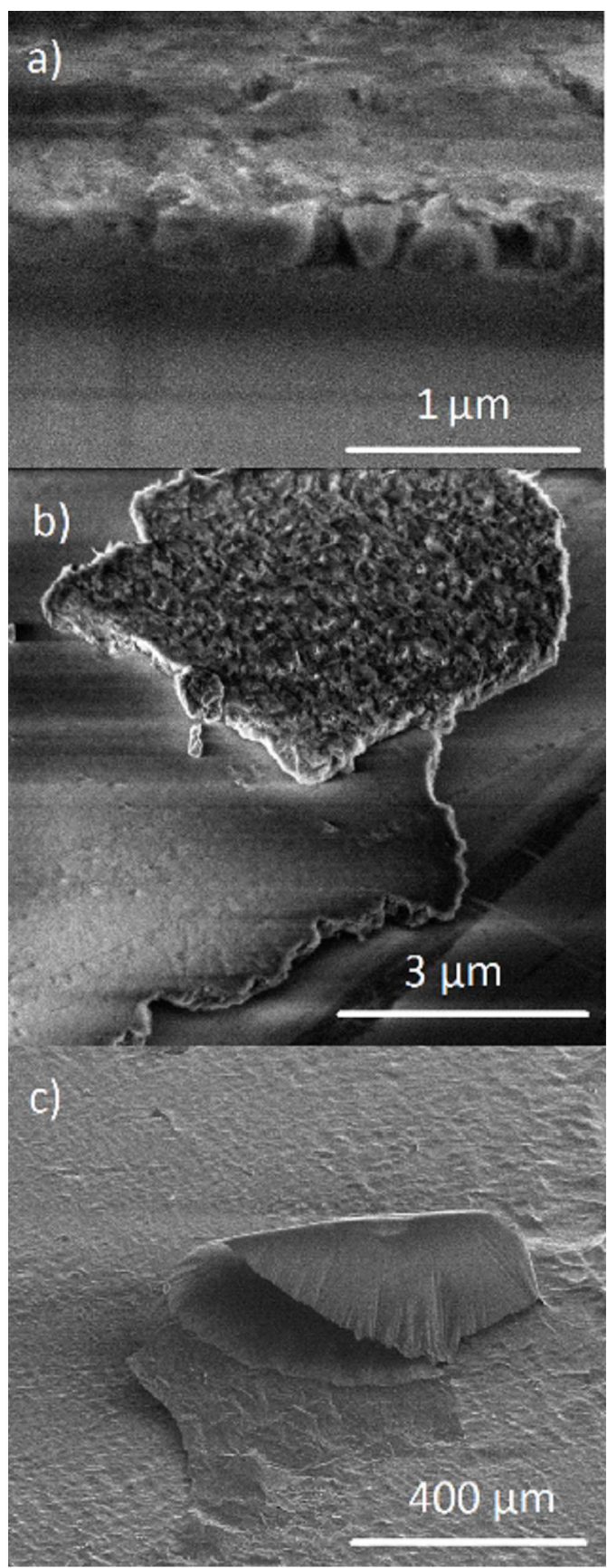

Fig. 1. Selected SEM picture: (a) edge-on view of BN layer on sapphire substrate. Grainy structure of the BN is visible. (b) BN layer mechanically scrapped off from sapphire substrate. Picture shows the substrate, BN layer and scrapped BN platelet. Traces of steel tweezers are visible on substrate and layer surface (c) BN layer spontaneously ex-foliated from GaN substrate and manually transferred to adhesive tape.

\subsection{Raman spectroscopy}

The crystallographic structure was confirmed by the Raman spectroscopy. A line near $1370 \mathrm{~cm}^{-1}$, characteristic for h-BN [4, 5] appeared (Fig. 2). Simultaneously there was no line at $1055 \mathrm{~cm}^{-1}$ that would be expected for $\mathrm{c}-\mathrm{BN}[4,5]$.

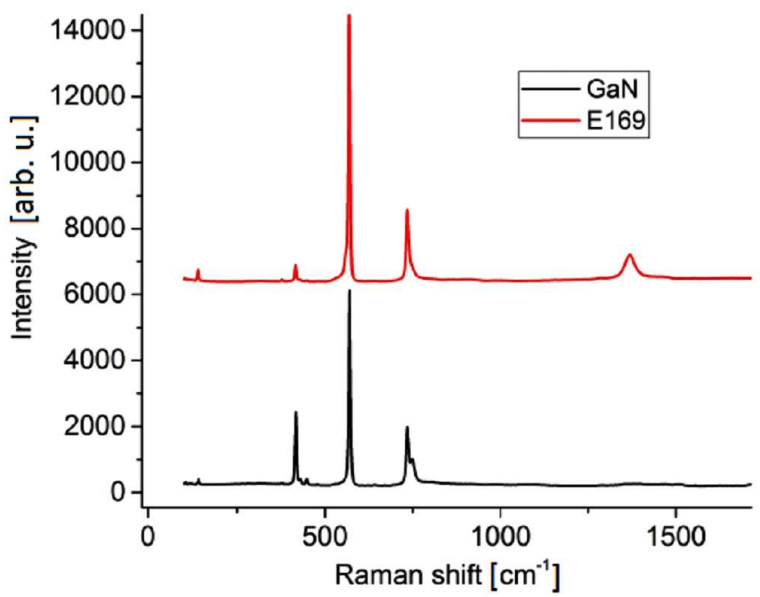

Fig. 2. The Raman spectra of the boron nitride on GaN (labeled E169) and reference gallium nitride samples. Line near the $1370 \mathrm{~cm}^{-1}$ is characteristic for h-BN.

\subsection{Absorption}

Absorption spectra were measured at room temperature.

As seen in Fig. 3, for thicker samples (grown at $1350^{\circ} \mathrm{C}$ ) the absorption edges started at around $5.6 \mathrm{eV}$. Since hexagonal BN band gap was reported at $5.96 \mathrm{eV} \mathrm{[6]}$ and cubic at $6.36 \mathrm{eV} \mathrm{[5],} \mathrm{so} \mathrm{we} \mathrm{can} \mathrm{expect} \mathrm{that} \mathrm{our} \mathrm{sam-}$ ples are of hexagonal phase. In all spectra a maximum at $6.1 \mathrm{eV}$ is observed. It could be related to the exciton absorption in hexagonal BN.

\subsection{Time resolved photoluminescence}

The photoluminescence was excited with $240 \mathrm{~nm}$ laser line $(5.16 \mathrm{eV})$.

It was too low for excitation of band-gap luminescence, however it revealed emission related to deep defects at $3.77 \mathrm{eV} \mathrm{[6]} \mathrm{and} 3.03 \mathrm{eV}$ (not shown). The luminescence decay (Fig. 4) was two-exponential with characteristic times of $\tau_{1}=0.16(1) \mathrm{ns}$ and $\tau_{2}=0.94(5) \mathrm{ns}$. Probably shorter time $\tau_{1}$ is due to diffusion of carriers and $\tau_{2}$ represents decay of trapped carriers.

\section{Conclusions}

Hexagonal boron nitride were successively grown in MOVPE system that was originally designed for GaN. Growth rate of $150 \mathrm{~nm}$ per hour was achieved. Layers had grainy structure characteristic for materials grown in too low temperature. Samples were characterized by SEM, AFM, the Raman spectroscopy, absorption and 


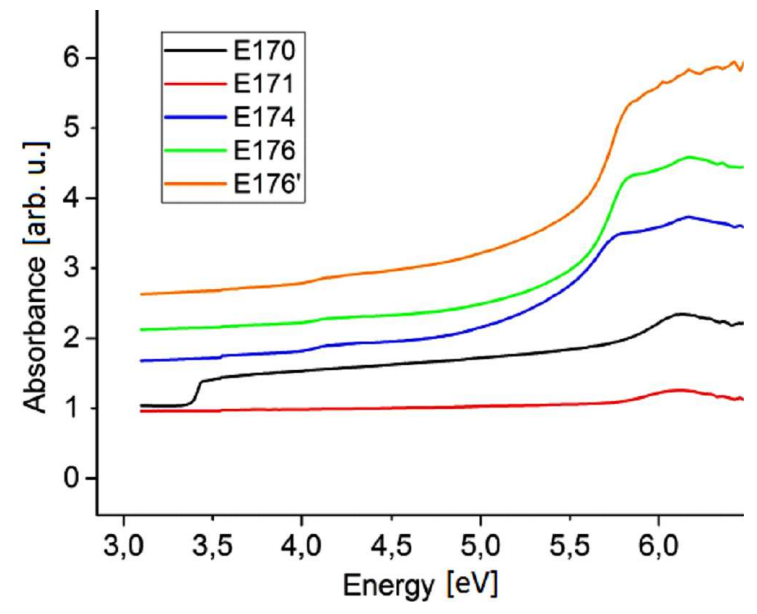

Fig. 3. The absorption spectra of BN samples. For E170 there is an additional absorption from $150 \mathrm{~nm} \mathrm{GaN}$ at about $3.4 \mathrm{eV}$.

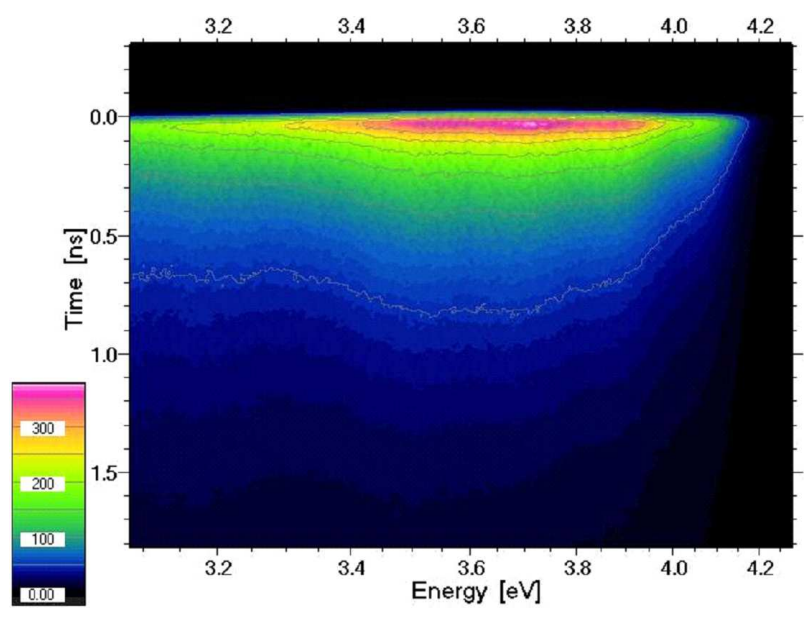

Fig. 4. Time resolved photoluminescence of the BN sample at room temperature.

time-resolved photoluminescence. The results confirmed that the obtained material was boron nitride in hexagonal phase of good optical quality. Choosing appropriate growth conditions (temperature, flow rate, initial structure under BN etc.) is crucial for achieving high quality crystals with good structural and optical properties.

\section{Acknowledgments}

The research was partially supported by the European Union within European Regional Development Funds, through the Innovative Economy Grant POIG.02.01.00$14-122 / 09$.

\section{References}

[1] Yong-Nian Xu, W.Y. Ching, Phys. Rev. B 44, 7787 (1991).

[2] K. Watanabe, T. Taniguchi, T. Niiyama, K. Miya, M. Taniguchi, Nature Photon. 3, 591 (2009).

[3] Y. Xu, Z. Guo, H. Chen, Y. Yuan, J. Lou, X. Lin, H. Gao, H. Chen, B. Yu , Appl. Phys. Lett. 99 , 133109 (2011).

[4] S. Reich, A.C. Ferrari, R. Arenal, A. Loiseau, I. Bello, J. Robertson, Phys. Rev. B 71, 205201 (2005).

[5] D.A. Evans, A.G. McGlynn, B.M. Towlson, M. Gunn, D. Jones, T.E. Jenkins, R. Winter, N.R.J. Poolton, J. Phys. Condens. Matter 20, 075233 (2008).

[6] M.G. Silly, P. Jaffrennou, J. Barjon, J.-S. Lauret, F. Ducastelle, A. Loiseau, E. Obraztsova, B. AttalTretout, E. Rosencher, Phys. Rev. B 75, 085205 (2007). 